\title{
Resveratrol alleviates sepsis-induced myocardial injury in rats by suppressing neutrophil accumulation, the induction of TNF- $\alpha$ and myocardial apoptosis via activation of Sirt1
}

\author{
RUI AN $^{1^{*}}$, LEI ZHAO ${ }^{2 *}$, JIAN XU $^{1 *}$, CONG XI ${ }^{3}$, HAIXUN LI ${ }^{1}$, \\ GUOHONG SHEN $^{4}$, WEI ZHANG ${ }^{1}$, SHUMIAO ZHANG ${ }^{5}$ and LIJUN SUN ${ }^{1}$
}

\begin{abstract}
Departments of ${ }^{1}$ Radiology and ${ }^{2}$ Neurosurgery, Xijing Hospital, The Fourth Military Medical University, Xi'an, Shaanxi 710032; ${ }^{3}$ Department of Neurology, Baoji City People's Hospital, Baoji, Shaanxi 721000;

${ }^{4}$ Integrated Branch, Armed Police Corps Hospital of Shaanxi Taiyuan, Shaanxi 030006;

${ }^{5}$ Department of Physiology, The Fourth Military Medical University, Xi'an, Shaanxi 710032, P.R. China
\end{abstract}

Received October 13, 2015; Accepted August 24, 2016

DOI: $10.3892 / \mathrm{mmr} .2016 .5861$

\begin{abstract}
Sepsis is a severe inflammatory response to systemic infection that frequently affects the myocardium. Previous studies have suggested that resveratrol (RESV) is protective in sepsis. The present study aimed to investigate the role of sirtuin 1 (Sirt1) signaling in the protective effect of intraperitoneally administered RESV against sepsis-induced myocardial injury. Cecal ligation and puncture, or a sham operation, were performed in male Sprague-Dawley rats, and the levels of tumor necrosis factor (TNF)- $\alpha$ and myeloperoxidase (MPO) were assessed by ELISA and an MPO activity kit, respectively. The extent of myocardial apoptosis was assessed by TUNEL staining. The protein expression levels of Sirt1, acetylated (Ac)-Forkhead box O1 (FoxO1), B cell lymphoma 2 apoptosis regulator $(\mathrm{Bcl}-2)$ and $\mathrm{Bcl}-2$ associated protein $\mathrm{X}$ apoptosis regulator (Bax) were detected by western blot analysis. RESV was demonstrated to attenuate myocardial apoptosis and decrease the production of TNF- $\alpha$ and MPO. Additionally, RESV upregulated the expression of Sirt1 and $\mathrm{Bcl}-2$, and downregulated the expression of Ac-FoxO1 and Bax. The protective effects of RESV were abolished by EX527, a Sirt1 inhibitor. RESV has therefore been demonstrated to attenuate myocardial injury in sepsis by decreasing neutrophil accumulation, TNF- $\alpha$ expression, and myocardial apoptosis via activation of Sirt1 signaling. These results suggest a novel therapeutic strategy for the clinical treatment of sepsis.
\end{abstract}

Correspondence to: Dr Lijun Sun, Department of Radiology, Xijing Hospital, The Fourth Military Medical University, 169 Changle West Road, Xi'an, Shaanxi 710032, P.R. China

E-mail: sunljfmmu@163.com

${ }^{*}$ Contributed equally

Key words: sepsis, cardiac damage, tumor necrosis factor- $\alpha$, apoptosis, sirtuin 1

\section{Introduction}

Sepsis, characterized by whole-body inflammation, is caused by a severe systemic infection (1). Sepsis is a leading cause of mortality in critically ill patients and the predominant cause of mortality in non-coronary intensive care units (2-4). The overproduction of cytokines that are induced by an infectious stimulus is the hallmark of sepsis, and leads to multiple organ dysfunction and consequently to a high mortality (5). Myocardial dysfunction is a recognized manifestation of this lethal condition (6-8).

Resveratrol (RESV) is a polyphenolic phytoalexin that has previously been suggested to exert cardioprotective effects $(9,10)$. It has been suggested to cause the 'French Paradox'; the low incidence of cardiovascular diseases in the French population despite a high consumption of wine and saturated fat (11). In addition, RESV is reported to be beneficial for sepsis-induced myocardial dysfunction (12). However, the underlying mechanism remains unclear.

Sirtuin 1 (Sirt1), a member of the silent mating type information regulator family of proteins, has multiple protective effects in various diseases (13-15). Sirtl suppresses cardiomyocyte apoptosis in diabetic cardiomyopathy (16) and is reported to suppress lung inflammasome activation in a murine model of sepsis (17). However, whether Sirtl activation is involved in the protective effect of RESV against sepsis-induced myocardial dysfunction remains unclear.

The current study used a rat model of sepsis involving cecal ligation and puncture (CLP) to investigate whether RESV was protective against sepsis-induced myocardial injury. In addition, the role of Sirt1 was evaluated in this model of CLP-induced sepsis.

\section{Materials and methods}

Animals and reagents. The present study was approved by the Committee for Animal Research of the Fourth Military Medical University (Xi'an, China). All animal experiments were performed in accordance with the National Institutes 
of Health Guidelines on the Use of Laboratory Animals (Bethesda, MD, USA). A total of 40 healthy, specific pathogen-free, male Sprague-Dawley rats (age, 3 months; weight, 200-250 g), were obtained from the Animal Center of the Fourth Military Medical University (Xi'an, China). Rats were housed in constant humidity $(50 \%)$ and temperature $\left(25 \pm 2^{\circ} \mathrm{C}\right)$ animal facilities with free access to standard laboratory food and water with a $12 \mathrm{~h}$ light/dark cycle.

RESV was purchased from Sigma-Aldrich (Merck Millipore, Darmstadt, Germany). The Sirt1 inhibitor, EX527, was purchased from Tocris Bioscience (Bristol, UK). Rabbit antibodies against Sirt1 (cat. no. 9475), acetylated-Forkhead box O1 (Ac-FoxO1; cat. no. 2880), B cell lymphoma 2 apoptosis regulator (Bcl-2; cat. no. 2870), and Bcl-2 associated protein $\mathrm{X}$ apoptosis regulator (Bax; cat. no. 2772) were purchased from Cell Signaling Technology, Inc. (Beverly, MA, USA). Antibodies against glyceraldehyde 3-phosphate dehydrogenase (GAPDH; cat. no. BM1623), and goat anti-rabbit (for Sirt1, Ac-FoxO1, Bcl-2 and Bax; cat. no. BA1039) and goat anti-mouse (for GAPDH; cat. no. BA1089) secondary antibodies were purchased from Wuhan Boster Biological Technology, Ltd. (Wuhan, China).

CLP surgery. To establish sepsis, rats were subjected to a CLP procedure. Animals were generally anesthetized with chloral hydrate (Sigma-Aldrich; Merck Millipore) $(350 \mathrm{mg} / \mathrm{kg}$ ) via intraperitoneal injection, and an abdominal midline incision (2-3 cm) was created to expose the cecum. The cecum was exteriorized and isolated, and a midpiece ligation of the cecum was made using 4-0 silk. The cecum was then perforated below the ligation twice with an 18-gauge needle, and a small amount of stool was extruded through the puncture holes to ensure patency. The cecum was then relocated to its normal intra-abdominal position and the abdomen was closed by suturing the muscle and skin. For the sham-operated animals, the cecum was isolated without ligation and puncturing. All animals received $0.9 \%$ saline solution $(40 \mathrm{ml} / \mathrm{kg}$ of body weight) subcutaneously immediately following the surgery and every subsequent $24 \mathrm{~h}$.

Experimental protocol. A total of 40 rats were randomly divided into 5 groups of 8 animals: Sham operation without CLP; CLP; CLP + RESV; CLP + RESV + EX527; and CLP + EX527. RESV was administered intraperitoneally at $60 \mathrm{mg} / \mathrm{kg}$ per rat, at 3, 12 and $24 \mathrm{~h}$ post-surgery. The Sirt1 inhibitor, EX527, was dissolved in dimethyl sulfoxide and diluted to the final concentration with normal saline. EX527, or the same volume of vehicle, was intraperitoneally injected at a dose of $5 \mathrm{mg} / \mathrm{kg}$ every two days, beginning 8 days prior to CLP surgery. A CLP + EX527 group was included to assess any contribution of EX527 to CLP-induced sepsis.

Hemodynamic assessment. Rats were anesthetized with chloral hydrate at $48 \mathrm{~h}$ post-CLP surgery, and pressure tracings of cardiac function were analyzed using an RM-6280 Multi-channel Physiological Signal Recording system (Chengdu Science Instrument Factory, Chengdu, China) for the assessment of cardiac function. A high-fidelity, pressure-transducing catheter, filled with heparinized saline, was inserted via the right carotid artery into the left ventricle.
When the rats returned to a stable condition, hemodynamic changes, including left ventricular systolic pressure (LVSP) and left ventricular end-diastolic pressure (LVEDP), and their first derivative with respect to time $\left(\mathrm{LV} \pm \mathrm{dP} / \mathrm{dt}_{\max }\right)$ were measured continuously (over 3 different periods, 10 cardiac cycles for each).

Myocardial apoptosis. Rats were anesthetized with chloral hydrate and sacrificed by thoracotomy. Hearts were harvested at $48 \mathrm{~h}$ post-CLP surgery and fixed in $4 \%$ paraformaldehyde for $48 \mathrm{~h}$. Following embedding in paraffin, $5 \mu \mathrm{m}$ thick sections of whole hearts were obtained. Terminal deoxynucleotidyl transferase dUTP nick-end labeling (TUNEL staining) was used to assess the number of apoptotic myocardial cells. TUNEL kits were purchased from Roche Diagnostics $\mathrm{GmbH}$ (Mannheim, Germany). TUNEL reaction mixture (50 $\mu \mathrm{l})$ was added to each sample and the slides incubated in a humidified atmosphere for $60 \mathrm{~min}$ at $37^{\circ} \mathrm{C}$ in the dark. Slides were rinsed with phosphate-buffered saline (PBS; $\mathrm{pH} 7.4$ ) three times, for 5 min each time. To detect the nuclei, slides were incubated with 4',6-diamidino-2-phenylindole (DAPI) for $5 \mathrm{~min}$ at room temperature in the dark, rinsed with PBS three times, for 5 min each time, and observed under a fluorescence microscope. TUNEL-positive nuclei were green and DAPI-positive nuclei were blue. The percentage of apoptotic nuclei (apoptotic nuclei/total nuclei x100) was calculated in 5 randomly chosen fields per slide $(3$ slides per section and 3 sections per rat, 26 rats in total).

Detection of tumor necrosis factor (TNF)- $\alpha$ in the serum and myocardial tissue. Blood samples were collected from the abdominal aorta at the time of sacrifice $48 \mathrm{~h}$ post-CLP surgery and centrifuged $(1,000 \times \mathrm{g}$ for $15 \mathrm{~min})$ to obtain serum that was stored at $-80^{\circ} \mathrm{C}$ prior to further analysis. Serum and heart samples, harvested and stored at $-80^{\circ} \mathrm{C}$ until analysis, were processed to measure TNF- $\alpha$ levels with an enzyme-linked immunosorbent assay (ELISA) kit (cat. no. JER-06; Joyee Biotechnics Co., Ltd., Shanghai, China), used according to the manufacturer's protocol.

Evaluation of myeloperoxidase (MPO) levels. Myocardial tissues were collected $48 \mathrm{~h}$ after CLP surgery. An MPO Activity assay kit (cat. no. A044; Nanjing Jiancheng Bioengineering Institute, Nanjing, China) was employed to detect the level of MPO in the myocardial tissue.

Western blotting. Left ventricular myocardial tissue was lysed in sample buffer (Pulse Yuan Biological Technology Co., Ltd., Xi'an, China), homogenized, and centrifuged at 10,000 x $g$ for $15 \mathrm{~min}$. A bicinchoninic acid assay was performed for protein quantification. Equal amounts of total protein $(40 \mu \mathrm{g})$ were separated on $10-15 \%$ denaturing gels by sodium dodecyl sulfate-polyacrylamide gel electrophoresis. The proteins were transferred onto nitrocellulose membranes (EMD Millipore, Billerica, MA, USA) and incubated in 10\% skimmed milk in Tris-buffered saline and $0.1 \%$ Tween-20 (TBST) for $2 \mathrm{~h}$. The membrane was then incubated with primary antibodies against Sirt1, Ac-FoxO1, Bcl-2, or Bax, diluted 1:1,000, at $4^{\circ} \mathrm{C}$ overnight. GAPDH was selected as the loading control. The blots were washed with TBST and incubated with the appropriate 

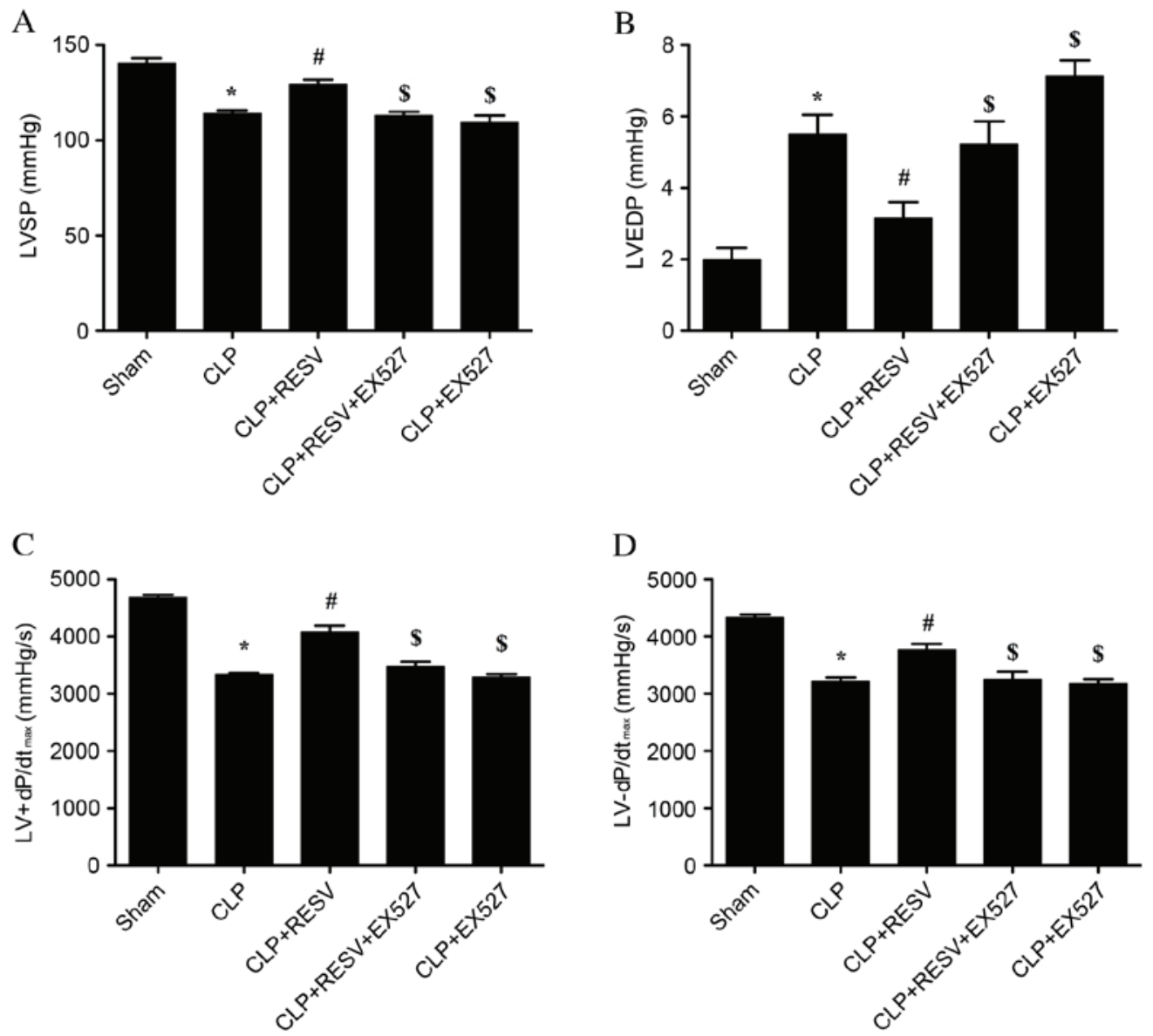

Figure 1. Invasive hemodynamic evaluation suggests that RESV improves cardiac function. (A) LVSP, (B) LVEDP, (C) LV+dP/dt ${ }_{\max }$ and (D) LV-dP/dt ${ }_{\max }$ were measured in rats following CLP and treatment with RESV, EX527 or both. Data are presented as the mean \pm standard error, $\mathrm{n}=8$ per group. $\mathrm{P}<0.05$ vs. sham group, ${ }^{\#} \mathrm{P}<0.05$ vs. CLP group, ${ }^{\$} \mathrm{P}<0.05$ vs. CLP + RESV group. LVSP, left ventricular systolic pressure; CLP, cecal ligation and puncture; RESV, resveratrol; LVEDP, left ventricular end diastolic pressure; $\mathrm{LV} \pm \mathrm{dP} / \mathrm{dt}_{\max }$, instantaneous first derivation of left ventricle pressure.

secondary antibodies conjugated to horseradish peroxidase diluted 1:5,000, for $1 \mathrm{~h}$ at room temperature, and then washed with TBST. Immunoreactive bands were detected using an enhanced chemiluminescence detection reagent (Beijing Solarbio Science \& Technology Co., Ltd., Beijing, China) using a Bio-Rad imaging system (Bio-Rad Laboratories, Inc., Hercules, CA, USA), and quantified using Quantity One software version 4.62 (Bio-Rad Laboratories, Inc.).

Statistical analysis. Data are presented as the mean \pm standard error. Significant differences among groups were evaluated by Student's $t$-test for unpaired data, or one-way analysis of variance followed by Dunnett's $t$-test for multiple comparisons. $\mathrm{P}<0.05$ was considered to indicate a statistically significant difference.

\section{Results}

RESV attenuates sepsis-induced myocardial injury in rats. CLP-induced sepsis resulted in a severe impairment in cardiovascular performance at $48 \mathrm{~h}$ post-CLP surgery. In the CLP group, the LVSP was significantly decreased $(\mathrm{P}<0.001$; Fig. 1A), the LVEDP was significantly elevated $(\mathrm{P}<0.001$; Fig. 1B), and $\mathrm{LV} \pm \mathrm{dP} / \mathrm{dt}_{\max }$ were significantly decreased compared with the sham group $(\mathrm{P}<0.001$ and $\mathrm{P}<0.001$, respectively; Fig. 1C and D). Treatment with RESV significantly prevented all of these detrimental effects induced by CLP ( $<<0.05$ CLP + RESV vs. CLP group; Fig. 1). EX527, a selective Sirt1 inhibitor, significantly attenuated the effects induced by RESV on LVSP, LVEDP and LV $\pm \mathrm{dP} / \mathrm{dt}_{\text {max }}(\mathrm{P}<0.05$, CLP + RESV + EX527 group vs. CLP + RESV group; Fig. 1). No significant difference was detected between the CLP and CLP+EX527 groups.

RESV reduces myocardial apoptosis in septic rats. The number of TUNEL-positive cells detected in myocardial tissue was increased in the CLP group compared with the sham group $(\mathrm{P}<0.001$; Fig. 2), indicating a significantly higher degree of apoptosis. There was a significant reduction in TUNEL-positive staining in the RESV-treated group compared with the CLP group ( $\mathrm{P}=0.0014$; Fig. 2), indicating an anti-apoptotic effect of RESV. EX527 suppressed the protective effect of RESV; increasing the apoptotic index in the CLP + RESV + EX527 group compared with the CLP + RESV group $(\mathrm{P}=0.0038$; Fig. 2). No significant difference was detected between the CLP and CLP+EX527 groups $(\mathrm{P}=0.9909)$.

RESV suppresses the sepsis-induced production of TNF- $\alpha$. An inflammatory response accompanied the CLP-induced sepsis. In the CLP group, the level of TNF- $\alpha$ was significantly 

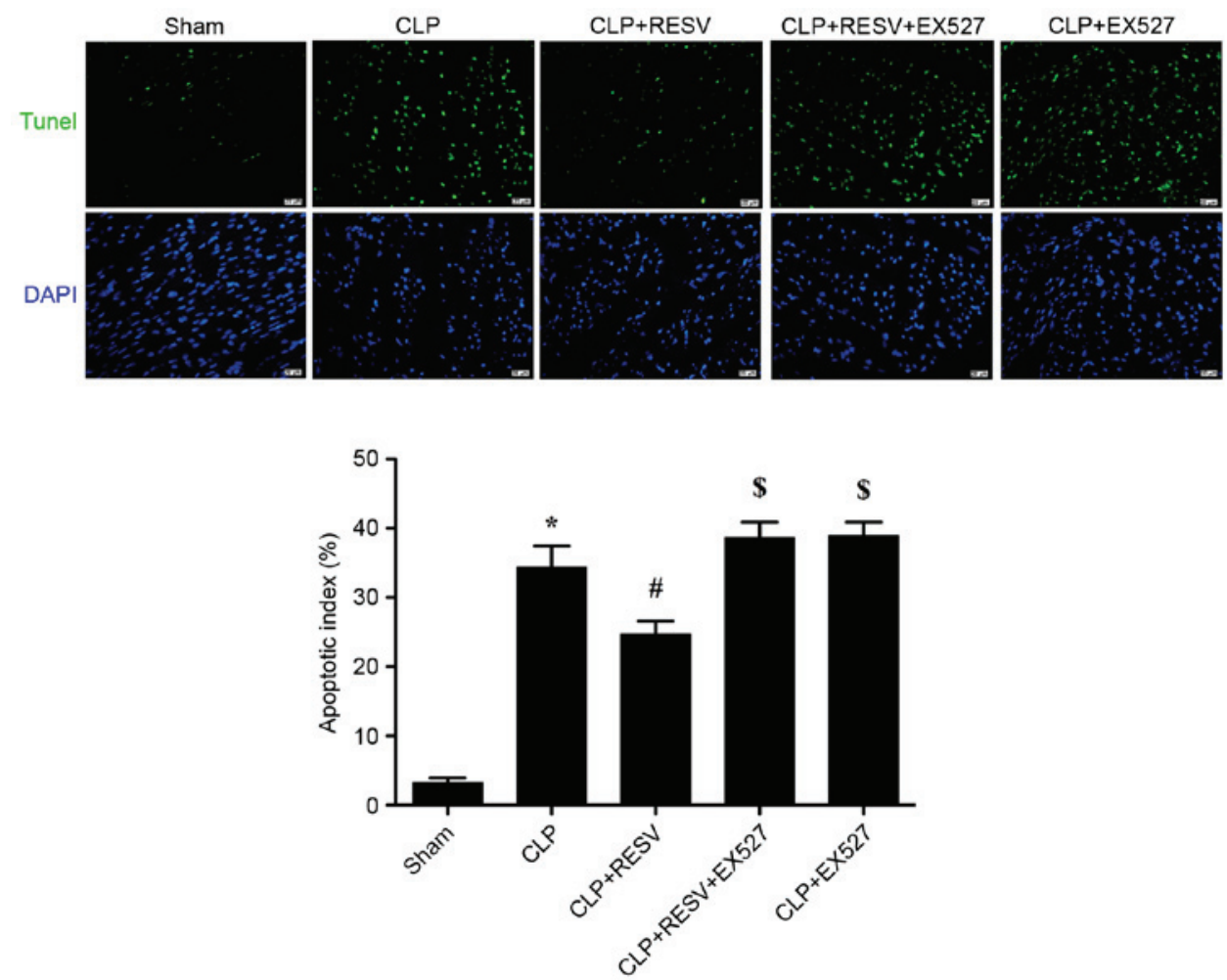

Figure 2. RESV suppression of sepsis-induced myocardial apoptosis is abolished by EX527. Apoptotic cells were detected by TUNEL (green), and the nuclei were detected by DAPI (blue). Representative images are shown. Magnification, $\mathrm{x} 400$. Scale bars $=20 \mu \mathrm{m}$. Data are presented as the mean \pm standard error, $\mathrm{n}=8$ per group. ${ }^{*} \mathrm{P}<0.05$ vs. sham group, ${ }^{\prime} \mathrm{P}<0.05$ vs. CLP group, ${ }^{\$} \mathrm{P}<0.05$ vs. CLP + RESV group. CLP, cecal ligation and puncture; RESV, resveratrol; TUNEL, terminal deoxynucleotidyl transferase dUTP nick-end labeling; DAPI, 4',6-diamidino-2-phenylindole.

increased in serum $(\mathrm{P}<0.001 ;$ Fig. $3 \mathrm{~A})$ and myocardial tissue compared with the sham group $(\mathrm{P}<0.001$; Fig. 3B). Treatment with RESV significantly decreased the levels of TNF- $\alpha$ in the CLP + RESV group compared with the CLP group in serum $(\mathrm{P}<0.001$; Fig. 3A) and myocardial tissue $(\mathrm{P}<0.001$; Fig. 3B). EX527 virtually eliminated the protective effect of RESV treatment in the CLP + RESV + EX527 group compared with the CLP + RESV group in serum $(\mathrm{P}<0.001$; Fig. $3 \mathrm{~A})$ and myocardial tissue $(\mathrm{P}<0.001$; Fig. $3 \mathrm{~B})$. No significant difference was detected between the CLP and CLP+EX527 groups $(\mathrm{P}=0.9992)$ in serum; however, in the myocardial tissue, EX527 significantly decreased the level of TNF- $\alpha$ compared with the CLP group $(\mathrm{P}=0.0008)$.

RESV inhibits neutrophil infiltration of myocardial tissue in septic rats. MPO, a heme protein predominantly expressed in neutrophils, is synthesized and stored in azurophilic granules of granulocytes and monocytes, and accounts for $5 \%$ of dry cell weight (18-20). The extent of neutrophil infiltration is partially reflected by the activity of MPO within a tissue. As demonstrated in Fig. 4, the activity of MPO in hearts from the CLP group was significantly increased compared with sham group $(\mathrm{P}=0.0004)$. By contrast, the activity of MPO was significantly reduced in the CLP + RESV group compared with the CLP group $(\mathrm{P}=0.0372)$. Furthermore, this effect was largely abolished by EX527 in the CLP + RESV + EX527 group compared with the CLP + RESV group $(\mathrm{P}=0.0130)$, indicating that the activation of Sirt1 may have an important role in the protective effect of RESV. No significant difference was detected between the CLP and CLP+EX527 groups $(\mathrm{P}=0.9878)$.
Expression and effect of Sirt1 in septic rats treated with RESV. To investigate the role of Sirt1 in injured myocardial tissue of rats with CLP-induced sepsis, the protein expression levels of Sirt1, Ac-FoxO1, Bcl-2 and Bax were assessed by western blot analysis (Fig. 5). The CLP group displayed significantly lower Sirt1 expression $(\mathrm{P}<0.001$; Fig. 5A), higher Ac-FoxO1 expression $(\mathrm{P}<0.001$; Fig. 5B), lower Bcl-2 expression $(\mathrm{P}<0.001$; Fig. $5 \mathrm{C})$ and higher Bax expression $(\mathrm{P}<0.001$; Fig. 5D) than the sham group. RESV prevented the CLP-induced downregulation of Sirt1 and Bcl-2 expression in the CLP + RESV group compared with the CLP group $(\mathrm{P}<0.001$ and $\mathrm{P}<0.001$, respectively; Fig. 5A and $\mathrm{C}$ ), and, similarly, prevented CLP-induced upregulation of Ac-FoxO1 and $\mathrm{Bax}(\mathrm{P}<0.001$ and $\mathrm{P}<0.001$, respectively; Fig. 5B and D). In the CLP + RESV+ EX527 group, all protective effects of RESV were prevented compared with the CLP + RESV group $(\mathrm{P}<0.05$; Fig. 5). No significant differences in Sirt1, Ac-FoxO1 and Bcl-2 were detected between the CLP and CLP+EX527 groups $(\mathrm{P}=0.9969, \mathrm{P}=0.5170$ and $\mathrm{P}=0.9936$, respectively); however, Bax was significantly decreased with the application of EX527 $(\mathrm{P}<0.001)$. This suggested that Sirt1 is important for the protective effect of RESV against CLP-induced myocardial injury.

\section{Discussion}

In the present study, a CLP-induced rat model of sepsis was established to mimic human sepsis, and used to investigate sepsis-induced cardiac dysfunction and the protective effect of RESV. Treatment with RESV was demonstrated to significantly suppress myocardial apoptosis, neutrophil 

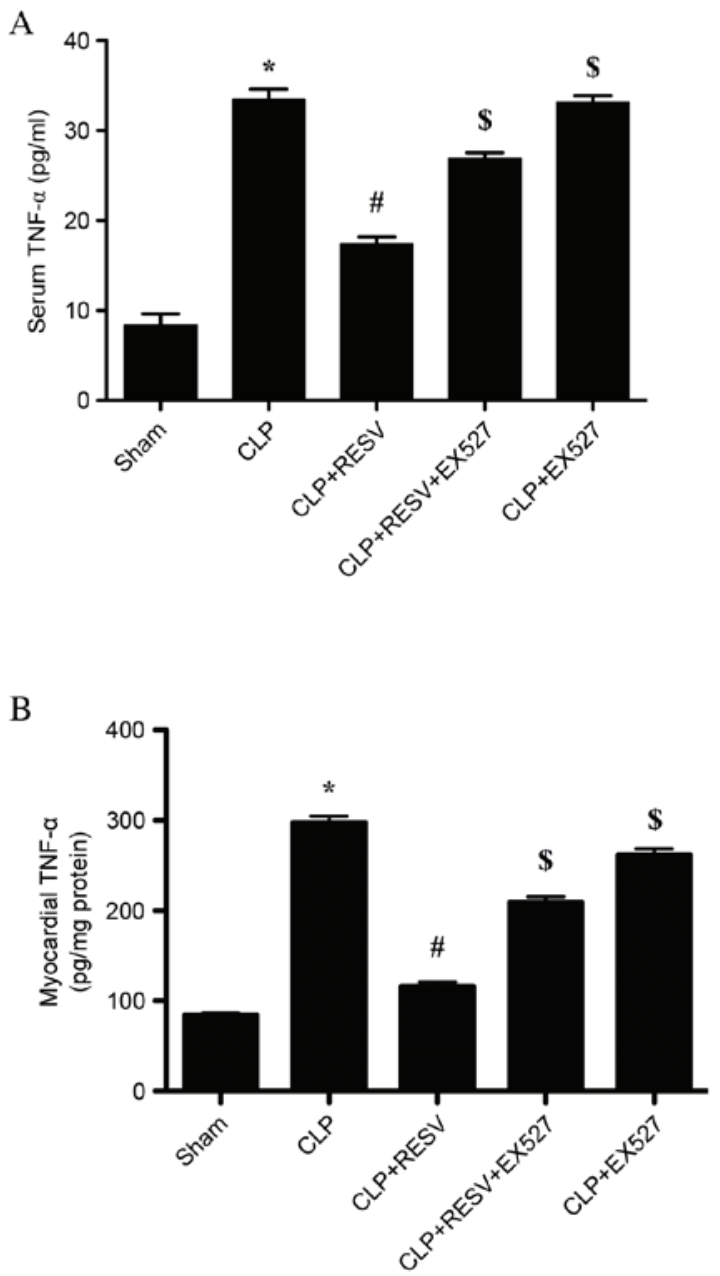

Figure 3. RESV decreases the levels of TNF- $\alpha$ in the serum and myocardial tissues in a CLP-induced sepsis model. The level of TNF- $\alpha$ in (A) serum and (B) myocardial tissue was determined by enzyme-linked immunosorbent assay. Data are presented as the mean \pm standard error, $n=8$ per group. ${ }^{*} \mathrm{P}<0.05$ vs. sham group, ${ }^{\text {"P }}<0.05$ vs. CLP group, ${ }^{~} \mathrm{P}<0.05$ vs. CLP + RESV group. TNF- $\alpha$, tumor necrosis factor $\alpha$; CLP, cecal ligation and puncture; RESV, resveratrol.

infiltration and TNF- $\alpha$ production during sepsis. Additionally, Sirtl was demonstrated to be involved in the cardioprotective effect of RESV.

Sepsis is a lethal condition characterized by a systemic inflammatory response syndrome, induced by a severe infection leading to multiple organ dysfunctions (21-23). The cardiovascular system is frequently affected by sepsis, and this effect has been studied for nearly six decades (23). Patients with sepsis and myocardial depression are at a 50-70\% greater risk of death than patients without cardiovascular complications (23), indicating that myocardial injury is an urgent problem to resolve. The underlying mechanisms of sepsis-induced myocardial dysfunction are thought to include metabolic changes (24), autonomic dysregulation (25), mitochondrial dysfunction (26), cell apoptosis (27), and inflammation (28). TNF- $\alpha$ is important during sepsis-induced inflammation (29). It has previously been reported that the application of murine monoclonal anti-TNF antibodies induced a transient improvement in ventricular function in patients with sepsis, suggesting that TNF may be involved in sepsis (30). Furthermore, TNF- $\alpha$ exerts a negative inotropic effect on the heart, leading to a decrease in blood

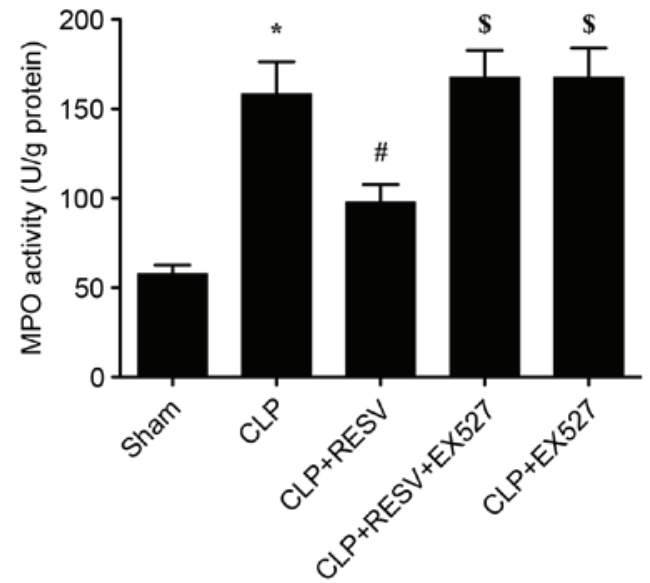

Figure 4. Effects of RESV and EX527 on sepsis-induced neutrophil infiltration. MPO activity was determined by enzyme-linked immunosorbent assay. Data are presented as the mean \pm standard error, $n=8$ per group. ${ }^{*} \mathrm{P}<0.05$ vs. sham group, ${ }^{\#} \mathrm{P}<0.05$ vs. CLP group,${ }^{\$} \mathrm{P}<0.05$ vs. CLP + RESV group. MPO, myeloperoxidase; CLP, cecal ligation and puncture; RESV, resveratrol.

pressure and cardiac output (29). TNF- $\alpha$ can also activate other inflammatory cells, such as neutrophils, triggering an inflammatory cascade (31). Thus, neutrophil infiltration is another factor associated with myocardial dysfunction induced by sepsis (32).

RESV, a natural phenolic anti-oxidant, has therapeutic benefits in sepsis (12,33-35). Recently, RESV was reported to attenuate microvascular inflammation in sepsis (36). Additionally, RESV was suggested to suppress high-mobility group protein box 1 nucleocytoplasmic translocation in sepsis-induced liver injury (13) and to alleviate sepsis-induced myocardial dysfunction via the Nrf2 transcription factor (35). In the present study, RESV was suggested to ameliorate myocardial dysfunction, suppress TNF- $\alpha$ activity in the serum and myocardium, and inhibit neutrophil accumulation in the myocardial tissue.

EX527 abolished the protective effect of RESV, indicating Sirtl activation is closely associated with the RESV mechanism of action. Sirt1, a nicotinamide adenine dinucleotide ${ }^{+}$-dependent class III histone deacetylase, is involved in numerous pathophysiological processes. Sirtl is essential for protein deacetylation and the regulation of pro-inflammatory cytokine release, apoptosis, stress resistance, metabolism, mitochondrial biogenesis, autophagy, senescence, differentiation and aging (9,37-39). The activation of Sirtl leads to the deacetylation and activation of FoxO, which promotes the synthesis of superoxide dismutase and catalase (40), therefore protecting the cell against oxidative stress. In addition, Sirt1 upregulates Bcl-2 expression and downregulates Bax expression, leading to an anti-apoptotic effect (41). In accordance with previous studies (42-44), the results of the present study suggest that RESV suppresses myocardial apoptosis by upregulating Bcl-2 expression and downregulating Bax expression. In addition, RESV promoted the deacetylation and activation of FoxO1, as evidenced by the decrease in Ac-FoxO1 detected by western blotting. However, the selective Sirt1 inhibitor, EX527, abolished the protective effects of RESV, indicating the involvement of Sirtl in this protective effect. The inclusion of the control group, CLP + EX527, throughout the study additionally excluded any contribution of EX527 itself to either the CLP-induced sepsis or the RESV-induced protections. 
A

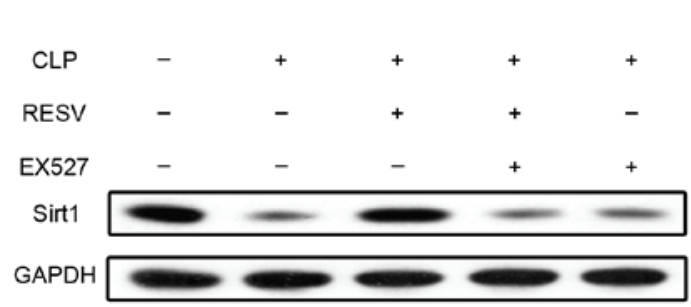

B

$\mathrm{C}$
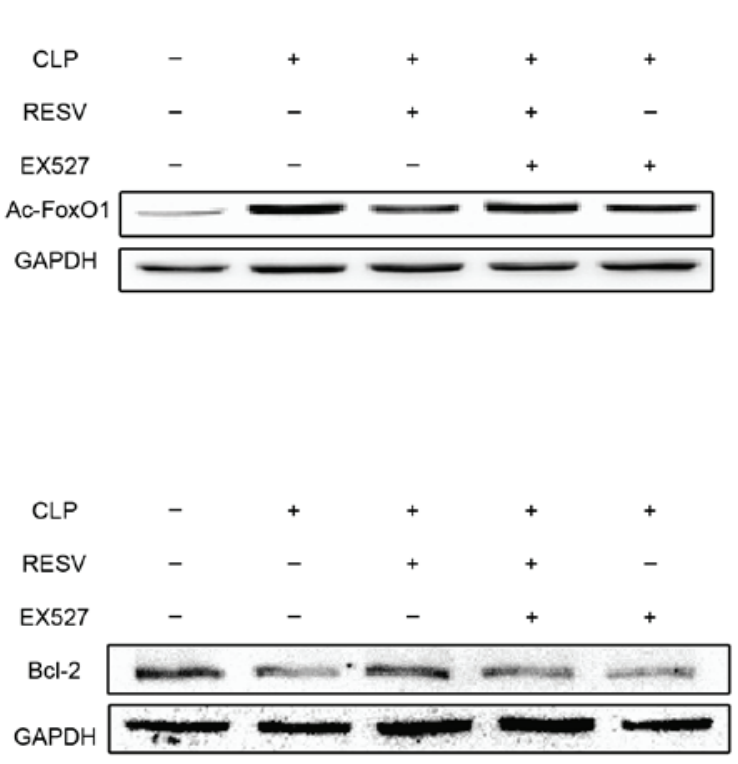

$\mathrm{D}$

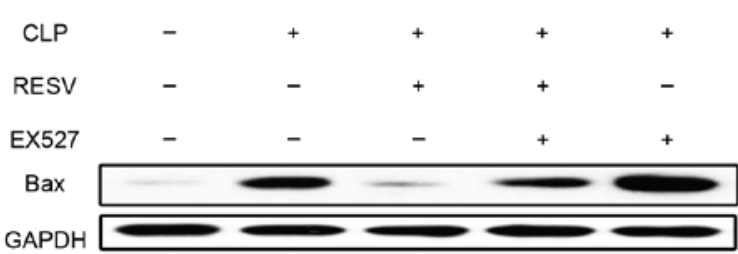

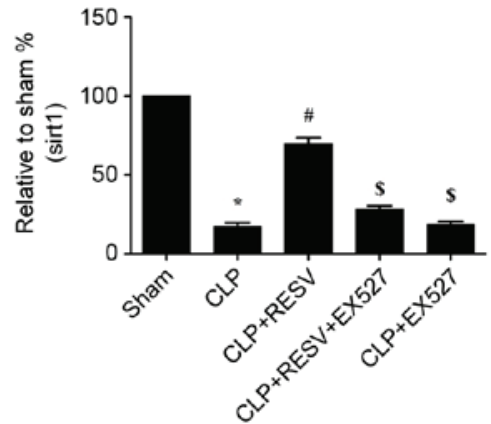
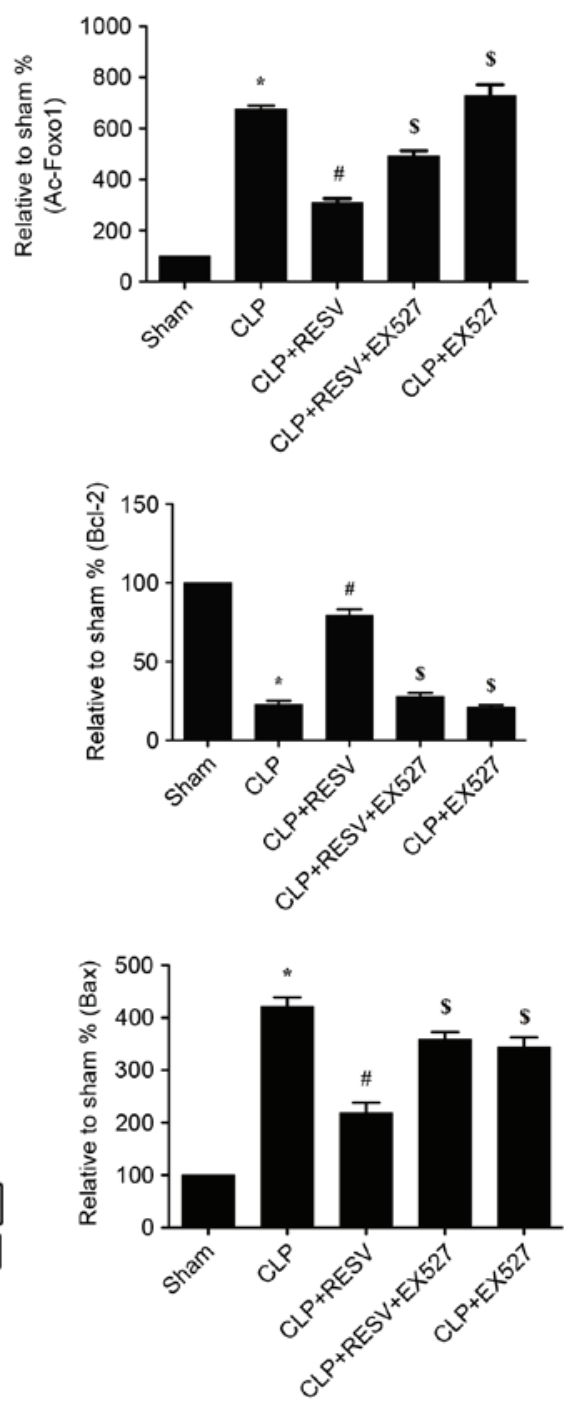

Figure 5. Effect of RESV on the protein expression levels of Sirt1, Ac-FoxO1, Bcl-2 and Bax following sepsis. Western blot analysis and quantification of the protein expression levels of (A) Sirt1, (B) Ac-FoxO1, (C) Bcl-2 and (D) Bax. Representative images of western blot results are shown. Quantification was relative to GAPDH. Data are presented as the mean \pm standard error, $n=8$ per group. ${ }^{*} \mathrm{P}<0.05$ vs. sham group, ${ }^{\prime} \mathrm{P}<0.05$ vs. CLP group, and ${ }^{\$} \mathrm{P}<0.05$ vs. CLP + RESV group. CLP, cecal ligation and puncture; RESV, resveratrol; Sirt1, sirtuin 1; GAPDH, glyceraldehyde 3-phosphate dehydrogenase; Ac-FoxO1, acetylated-Forkhead box O1, Bcl-2, B cell lymphoma 2 apoptosis regulator; Bax, Bcl-2 associated protein X apoptosis regulator.

In conclusion, the results of the present study demonstrate that RESV ameliorates cardiac dysfunction and apoptosis induced by sepsis, and suppresses TNF- $\alpha$ production and neutrophil accumulation. Additionally, activation of Sirt1 signaling is involved in the protective effects of RESV. The present study, therefore, provides evidence supporting further investigations into the clinical use of RESV in the treatment of sepsis-induced myocardial injury.

\section{Acknowledgements}

The present study was supported by grants from the National Natural Science Foundation of China (grant no. 81170185).

\section{References}

1. Annane D, Bellissant E and Cavaillon JM: Septic shock. Lancet 365: 63-78, 2005. 
2. Frolkis I, Klein Y, Locker C, Adi N, Dahan E, Uretzsky G, Shapira I and Sorkine P: Vipera aspis venom reduces lethality and down-regulates tumor necrosis factor-alpha in a rat model of LPS-induced sepsis. Cytokine 49: 319-324, 2010.

3. Angus DC, Linde-Zwirble WT, Lidicker J, Clermont G, Carcillo J and Pinsky MR: Epidemiology of severe sepsis in the United States: Analysis of incidence, outcome, and associated costs of care. Crit Care Med 29: 1303-1310, 2001.

4. Dellinger RP, Levy MM, Rhodes A, Annane D, Gerlach H, Opal SM, Sevransky JE, Sprung CL, Douglas IS, Jaeschke R, et al: Surviving sepsis campaign: International guidelines for management of severe sepsis and septic shock, 2012. Intensive Care Med 39: 165-228, 2013

5. An R, Zhao L, Xi C, Li H, Shen G, Liu H, Zhang S and Sun L: Melatonin attenuates sepsis-induced cardiac dysfunction via a PI3K/Akt-dependent mechanism. Basic Res Cardiol 111: 8, 2016

6. Rudiger A and Singer M: Mechanisms of sepsis-induced cardiac dysfunction. Crit Care Med 35: 1599-1608, 2007.

7. Court O, Kumar A, Parrillo JE and Kumar A: Clinical review: Myocardial depression in sepsis and septic shock. Crit Care 6 : 500-508, 2002

8. van der Poll T and van Deventer SJ: Cytokines and anticytokines in the pathogenesis of sepsis. Infect Dis Clin North Am 13: 413-426, ix, 1999.

9. Borra MT, Smith BC and Denu JM: Mechanism of human SIRT1 activation by resveratrol. J Biol Chem 280: 17187-17195, 2005.

10. Li YG, Zhu W, Tao JP, Xin P, Liu MY, Li JB and Wei M Resveratrol protects cardiomyocytes from oxidative stress through SIRT1 and mitochondrial biogenesis signaling pathways. Biochem Biophys Res Commun 438: 270-276, 2013.

11. Liu BL, Zhang X, Zhang W and Zhen HN: New enlightenment of French Paradox: Resveratrol's potential for cancer chemoprevention and anti-cancer therapy. Cancer Biol Ther 6: 1833-1836, 2007.

12. Smeding L, Leong-Poi H, Hu P, Shan Y, Haitsma JJ, Horvath E, Furmli S, Masoom H, Kuiper JW, Slutsky AS, et al: Salutary effect of resveratrol on sepsis-induced myocardial depression. Crit Care Med 40: 1896-1907, 2012.

13. Xu W, Lu Y, Yao J, Li Z, Chen Z, Wang G, Jing H, Zhang X, Li M, Peng J and Tian X: Novel role of resveratrol: Suppression of high-mobility group protein box 1 nucleocytoplasmic translocation by the upregulation of sirtuin 1 in sepsis-induced liver injury. Shock 42: 440-447, 2014

14. Xu F, Burk D, Gao Z, Yin J, Zhang X, Weng J and Ye J: Angiogenic deficiency and adipose tissue dysfunction are associated with macrophage malfunction in SIRT1-/- mice. Endocrinology 153: $1706-1716,2012$

15. Motta MC, Divecha N, Lemieux M, Kamel C, Chen D, Gu W, Bultsma Y, McBurney M and Guarente L: Mammalian SIRT1 represses forkhead transcription factors. Cell 116: 551-563, 2004

16. Guo R, Liu W, Liu B, Zhang B, Li W and Xu Y: SIRT1 suppresses cardiomyocyte apoptosis in diabetic cardiomyopathy: An insight into endoplasmic reticulum stress response mechanism. Int J Cardiol 191: 36-45, 2015.

17. Gao R, Ma Z, Hu Y, Chen J, Shetty S and Fu J: Sirt1 restrains lung inflammasome activation in a murine model of sepsis. Am J Physiol Lung Cell Mol Physiol 308: L847-L853, 2015.

18. Arnhold J: Properties, functions, and secretion of human myeloperoxidase. Biochemistry (Mosc) 69: 4-9, 2004.

19. Thom SR, Milovanova TN, Bogush M, Yang M, Bhopale VM, Pollock NW, Ljubkovic M, Denoble P, Madden D, Lozo M and Dujic Z: Bubbles, microparticles, and neutrophil activation: Changes with exercise level and breathing gas during open-water SCUBA diving. J Appl Physiol (1985) 114: 1396-1405, 2013.

20. Mallat Z, Hugel B, Ohan J, Lesèche G, Freyssinet JM and Tedgui A: Shed membrane microparticles with procoagulant potential in human atherosclerotic plaques: A role for apoptosis in plaque thrombogenicity. Circulation 99: 348-353, 1999.

21. Angus DC and van der Poll T: Severe sepsis and septic shock. N Engl J Med 369: 840-851, 2013.

22. Calis J, van Woensel $\mathrm{J}$ and Lemson $\mathrm{J}$ : Severe sepsis and septic shock. N Engl J Med 369: 2062, 2013

23. Merx MW and Weber C: Sepsis and the heart. Circulation 116 793-802, 2007.

24. Stanley WC, Recchia FA and Lopaschuk GD: Myocardial substrate metabolism in the normal and failing heart. Physiol Rev 85: 1093-1129, 2005.

25. Sharshar T, Gray F, Lorin de la Grandmaison G, Hopkinson NS, Ross E, Dorandeu A, Orlikowski D, Raphael JC, Gajdos P and Annane D: Apoptosis of neurons in cardiovascular autonomic centres triggered by inducible nitric oxide synthase after death from septic shock. Lancet 362: 1799-1805, 2003.
26. Wang X, Qin W, Qiu X, Cao J, Liu D and Sun B: A novel role of exogenous carbon monoxide on protecting cardiac function and improving survival against sepsis via mitochondrial energetic metabolism pathway. Int J Biol Sci 10: 777-788, 2014.

27. Zou X, Xu J, Yao S, Li J, Yang Y and Yang L: Endoplasmic reticulum stress-mediated autophagy protects against lipopolysaccharide-induced apoptosis in HL-1 cardiomyocytes. Exp Physiol 99: 1348-1358, 2014.

28. Zhang T, Yan T, Du J, Wang S and Yang H: Apigenin attenuates heart injury in lipopolysaccharide-induced endotoxemic model by suppressing sphingosine kinase 1/sphingosine 1-phosphate signaling pathway. Chem Biol Interact 233: 46-55, 2015.

29. Antonucci E, Fiaccadori E, Donadello K, Taccone FS, Franchi F and Scolletta S: Myocardial depression in sepsis: From pathogenesis to clinical manifestations and treatment. J Crit Care 29: 500-511, 2014

30. Vincent JL, Bakker J, Marécaux G, Schandene L, Kahn RJ and Dupont E: Administration of anti-TNF antibody improves left ventricular function in septic shock patients. Results of a pilot study. Chest 101: 810-815, 1992.

31. Wu X, Zhang B, Fan R, Zhao L, Wang Y, Zhang S, Kaye AD, Huang $L$ and Pei J: U50, 488H inhibits neutrophil accumulation and TNF- $\alpha$ induction induced by ischemia-reperfusion in rat heart. Cytokine 56: 503-507, 2011.

32. Unnewehr H, Rittirsch D, Sarma JV, Zetoune F, Flierl MA, Perl M, Denk S, Weiss M, Schneider ME, Monk PN, et al: Changes and regulation of the $\mathrm{C} 5 \mathrm{a}$ receptor on neutrophils during septic shock in humans. J Immunol 190: 4215-4225, 2013.

33. Kolgazi M, Sener G, Cetinel S, Gedik N and Alican I: Resveratrol reduces renal and lung injury caused by sepsis in rats. J Surg Res 134: 315-321, 2006 .

34. Holthoff JH, Wang Z, Seely KA, Gokden N and Mayeux PR: Resveratrol improves renal microcirculation, protects the tubular epithelium, and prolongs survival in a mouse model of sepsis-induced acute kidney injury. Kidney Int 81: 370-378, 2012.

35. Hao E, Lang F, Chen Y, Zhang H, Cong X, Shen X and Su G: Resveratrol alleviates endotoxin-induced myocardial toxicity via the Nrf2 transcription factor. PLoS One 8: e69452, 2013.

36. Wang X, BuechlerNL, Yoza BK, McCallCE and Vachharajani VT: Resveratrol attenuates microvascular inflammation in sepsis via SIRT-1-Induced modulation of adhesion molecules in ob/ob mice. Obesity (Silver Spring) 23: 1209-1217, 2015.

37. Bishayee A, Waghray A, Barnes KF, Mbimba T, Bhatia D, Chatterjee $M$ and Darvesh AS: Suppression of the inflammatory cascade is implicated in resveratrol chemoprevention of experimental hepatocarcinogenesis. Pharm Res 27: 1080-1091, 2010.

38. Lagouge M, Argmann C, Gerhart-Hines Z, Meziane H, Lerin C, Daussin F, Messadeq N, Milne J, Lambert P, Elliott P, et al: Resveratrol improves mitochondrial function and protects against metabolic disease by activating SIRT1 and PGC-1alpha. Cell 127: 1109-1122, 2006.

39. Sun W, Wang W, Kim J, Keng P, Yang S, Zhang H, Liu C, Okunieff $\mathrm{P}$ and Zhang L: Anti-cancer effect of resveratrol is associated with induction of apoptosis via a mitochondrial pathway alignment. Adv Exp Med Biol 614: 179-186, 2008.

40. Daitoku H, Hatta M, Matsuzaki H, Aratani S, Ohshima T, Miyagishi M, Nakajima T and Fukamizu A: Silent information regulator 2 potentiates Foxol-mediated transcription through its deacetylase activity. Proc Natl Acad Sci USA 101: 10042-10047, 2004.

41. Kalle AM, Mallika A, Badiger J, Alinakhi, Talukdar P and Sachchidanand: Inhibition of SIRT1 by a small molecule induces apoptosis in breast cancer cells. Biochem Biophys Res Commun 401: 13-19, 2010.

42. Guo S, Yao Q, Ke Z, Chen $\mathrm{H}$, Wu J and Liu C: Resveratrol attenuates high glucose-induced oxidative stress and cardiomyocyte apoptosis through AMPK. Mol Cell Endocrinol 412: 85-94, 2015.

43. Wang R, Liu YY, Liu XY, Jia SW, Zhao J, Cui D and Wang L: Resveratrol protects neurons and the myocardium by reducing oxidative stress and ameliorating mitochondria damage in a cerebral ischemia rat model. Cell Physiol Biochem 34: 854-864, 2014.

44. Lin Y, Zhu J, Zhang X, Wang J, Xiao W, Li B, Jin L, Lian J, Zhou L and Liu J: Inhibition of cardiomyocytes hypertrophy by resveratrol is Associated with Amelioration of endoplasmic reticulum stress. Cell Physiol Biochem 39: 780-789, 2016. 